\title{
Designing isoelectronic counterparts to layered group V semiconductors
}

\author{
Zhen Zhu, Jie Guan, Dan Liu, and David Tománel* \\ Physics and Astronomy Department, Michigan State University, East Lansing, Michigan 48824, USA
}

\begin{abstract}
In analogy to III-V compounds, which have significantly broadened the scope of group IV semiconductors, we propose IV-VI compounds as isoelectronic counterparts to layered group V semiconductors. Using $a b$ initio density functional theory, we study yet unrealized structural phases of silicon mono-sulfide (SiS). We find the black-phosphorus-like $\alpha$-SiS to be almost equally stable as the blue-phosphorus-like $\beta$-SiS. Both $\alpha$-SiS and $\beta$-SiS monolayers display a significant, indirect band gap that depends sensitively on the in-layer strain. Unlike 2D semiconductors of group V elements with the corresponding nonplanar structure, different SiS allotropes show a strong polarization either within or normal to the layers. We find that SiS may form both lateral and vertical heterostructures with phosphorene at a very small energy penalty, offering an unprecedented tunability in structural and electronic properties of SiS-P compounds.
\end{abstract}

\section{INTRODUCTION}

2D semiconductors of group $\mathrm{V}$ elements, including phosphorene and arsenene, have been rapidly attracting interest due to their significant fundamental band gap, large density of states near the Fermi level, and high and anisotropic carrier mobility[1]4]. Combination of these properties places these systems very favorably in the group of contenders for 2D electronics applications beyond graphene [5, 6] and transition metal dichalcogenides [7. Keeping in mind that the scope of group IV semiconductors such as $\mathrm{Si}$ has been broadened significantly by introducing isoelectronic III-V compounds, it is intriguing to see, whether the same can be achieved in IV-VI compounds that are isoelectronic to group V elemental semiconductors. Even though this specific point of view has not yet received attention, there has been interest in specific IV-VI compounds, such as $\mathrm{SnS}$, SnSe and GeTe, for thermoelectric and photovoltaic applications. 8 10, It appears likely that specific search for isoelectronic counterparts of layered semiconductors such as phosphorene and arsenene may guide us to yet unexplored 2D semiconducting IV-VI compounds that are stable, flexible, and display a tunable band gap.

As a yet unexplored IV-VI compound, we study the layered structure of silicon mono-sulfide ( $\mathrm{SiS})$. We use $a b$ initio density functional theory (DFT) to identify stable allotropes, determine their equilibrium geometry and electronic structure. We have identified two nearly equally stable allotropes, namely the black-phosphoruslike $\alpha$-SiS and the blue-phosphorus-like $\beta$-SiS, and show their structure in Fig. 11(a) and (d). Both $\alpha$-SiS and $\beta$ SiS monolayers display a significant, indirect band gap that depends sensitively on the in-layer strain. Unlike $2 \mathrm{D}$ semiconductors of group $\mathrm{V}$ elements with the corresponding nonplanar structure, different $\mathrm{SiS}$ allotropes show a strong polarization either within or normal to the layers. We find that SiS may form both lateral and vertical heterostructures with phosphorene at a very small energy penalty, offering an unprecedented tunability in structural and electronic properties of SiS-P compounds.

\section{RESULTS AND DISCUSSION}

Since all atoms in $s p^{3}$ layered structures of group V elements are threefold coordinated, the different allotropes can all be topologically mapped onto the honeycomb lattice of graphene with 2 sites per unit cell.[11] An easy way to generate IV-VI compounds that are isoelectronic to group $\mathrm{V}$ monolayers is to occupy one of these sites by a group IV and the other by a group VI element. In this way, we have generated the orthorhombic $\alpha$-SiS monolayer structure, shown in Fig. 11(a), from a monolayer of black phosphorene (or $\alpha$-P). The hexagonal $\beta$-SiS monolayer, shown in Fig. 1.(d), has been generated in the same way from the blue phosphorene (or $\beta-\mathrm{P}$ ) structure.

The monolayer structures have been optimized using DFT with the Perdew-Burke-Ernzerhof (PBE) 12 ] exchange-correlation functional, as discussed in the Methods Section. We found that presence of two elements with different local bonding preferences increases the thickness of the SiS monolayers when compared to the phosphorene counterparts. The 2D lattice of $\alpha$ $\mathrm{SiS}$ is spanned by the orthogonal Bravais lattice vectors $\left|\vec{a}_{1}\right|=4.76 \AA$ and $\left|\vec{a}_{2}\right|=3.40 \AA$, which are about $3 \%$ longer than the lattice vectors of black phosphorene. The $2 \mathrm{D}$ hexagonal lattice of $\beta$-SiS is spanned by two Bravais lattice vectors $a=\left|\vec{a}_{1}\right|=\left|\vec{a}_{2}\right|=3.35 \AA$, which are $\lesssim 1 \%$ longer than those of blue phosphorene. Unlike in the counterpart structures of the phosphorene monolayer, we find $\beta$-SiS to be more stable by about $12 \mathrm{meV} /$ atom than $\alpha$-SiS. We checked the phonon spectrum of free-standing $\alpha$-SiS and $\beta$-SiS monolayers and found no soft modes that would cause a spontaneous collapse of the structure.

An apparently different layered SiS structure had been synthesized [13] by exposing the layered $\mathrm{CaSi}_{2}$ structure to $\mathrm{S}_{2} \mathrm{Cl}_{2}$. It appears that the $\mathrm{S}_{2} \mathrm{Cl}_{2}$ reagent reacted with the $\mathrm{Ca}$ atoms intercalated between silicene layers in $\mathrm{CaSi}_{2}$ by forming $\mathrm{CaCl}_{2}$ and saturating the valencies of the silicene layers by $-\mathrm{S}-\mathrm{S}$ bridges. Even though no structural information has been provided in that study, we identified a likely candidate structure and found it to be less stable than the postulated SiS allotropes, on 

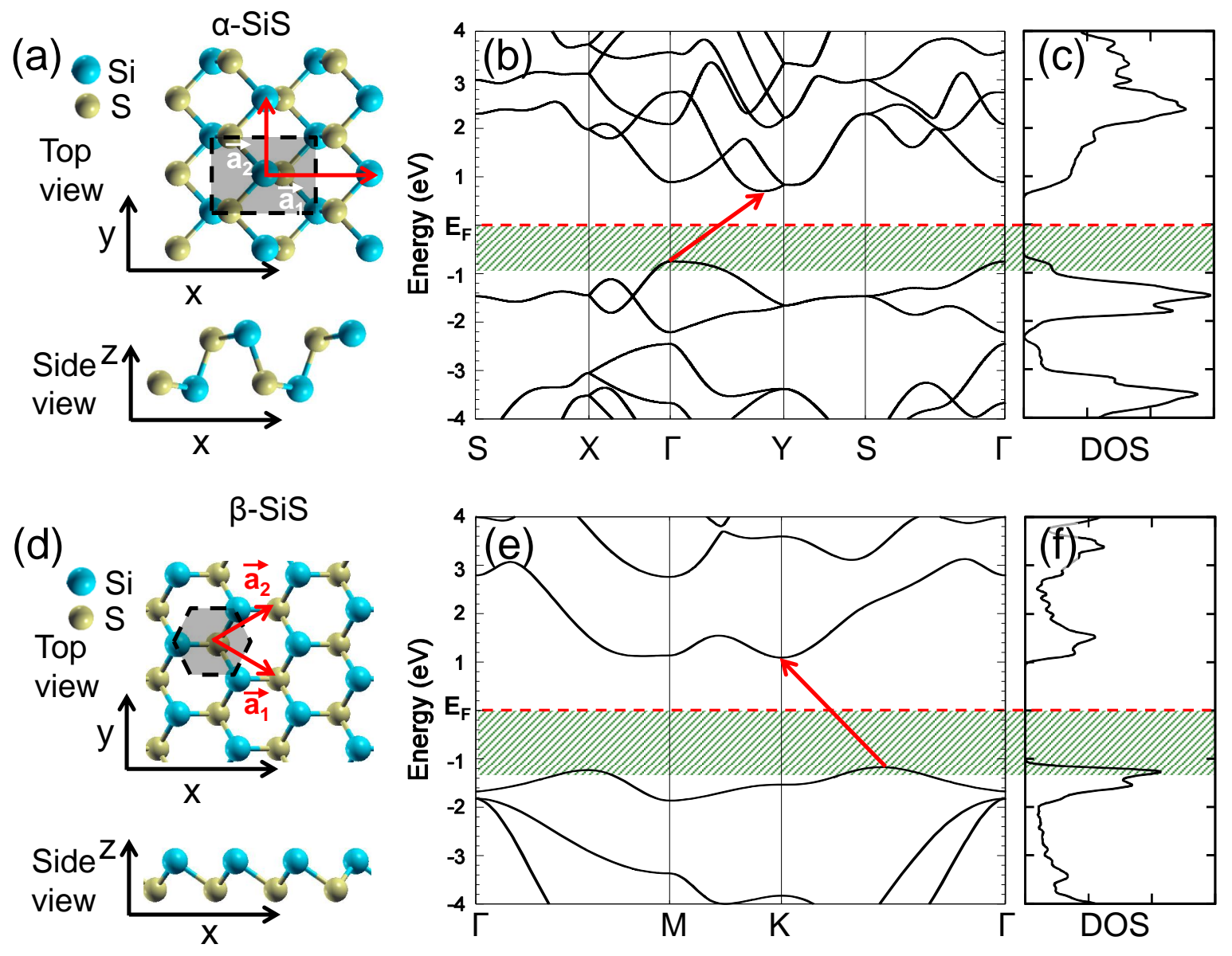

FIG. 1. (Color online) Atomic and electronic structure of [(a) to (c)] $\alpha$-SiS and [(d) to (f)] $\beta$-SiS monolayers. [(a) and (d)] Ball-and-stick models of the geometry, with S and Si atoms distinguished by size and color and the Wigner-Seitz cell indicated by the shaded region. [(b) and (e)] The electronic band structure and [(c) and (f)] the electronic density of states (DOS) of the systems. The energy range between $E_{F}$ and $0.2 \mathrm{eV}$ below the top of the valence band, indicated by the green shading, is used to identify valence frontier states.

which we focus next.

Since the electronegativity of $\mathrm{S}$ is higher than that of $\mathrm{Si}$, we expect an electron transfer from $\mathrm{Si}$ to $\mathrm{S}$ atoms. Based on a Mulliken population analysis, we estimate a net transfer of 0.3 electrons in $\alpha$-SiS and 0.2 electrons in $\beta$-SiS from silicon to sulfur atoms. This charge redistribution, combined with the nonplanarity of the structure, causes a net polarization. We find an in-plane polarization for $\alpha$-SiS and and an out-of-plane polarization for $\beta$-SiS.

Whereas DFT generally provides an accurate description of the total charge density and equilibrium geometry, interpretation of Kohn-Sham energy eigenvalues as quasiparticle energies is more problematic. Still, we present our DFT-PBE results for the electronic structure of $\alpha$ $\mathrm{SiS}$ and $\beta$-SiS monolayers in Fig. 1. Even though the fundamental band gaps are typically underestimated in this approach, the prediction that both systems are indirectgap semiconductors is likely correct. Our calculated band structure and the corresponding density of states for $\alpha$ -
SiS, presented in Fig. 1)(b,c), suggest that the fundamental band gap value $E_{g}=1.44 \mathrm{eV}$ should be significantly larger than in the isoelectronic black phosphorene counterpart. The band structure near the top of the valence band shows a significant anisotropy when comparing the $\Gamma-X$ and $\Gamma-Y$ directions. In analogy to phosphorene, $\alpha$-SiS should exhibit a higher hole mobility along the $x$-direction than along the $y$-direction.

The DFT-based fundamental band gap $E_{g}=2.26 \mathrm{eV}$ in the monolayer of $\beta$-SiS is even larger. The band structure in the symmetric honeycomb lattice of $\beta$-SiS is rather isotropic, as seen in Fig. 1(e). The top of the valence band is very flat, resulting in a heavy hole mass and a large density of states (DOS) in that region, as seen in Fig. 1(f).

The character of frontier states is of interest not only for a microscopic understanding of the conduction channels, but is also crucial for the design of optimum contacts. 14] Whereas DFT-based band gaps are typically underestimated as mentioned above, the electronic 

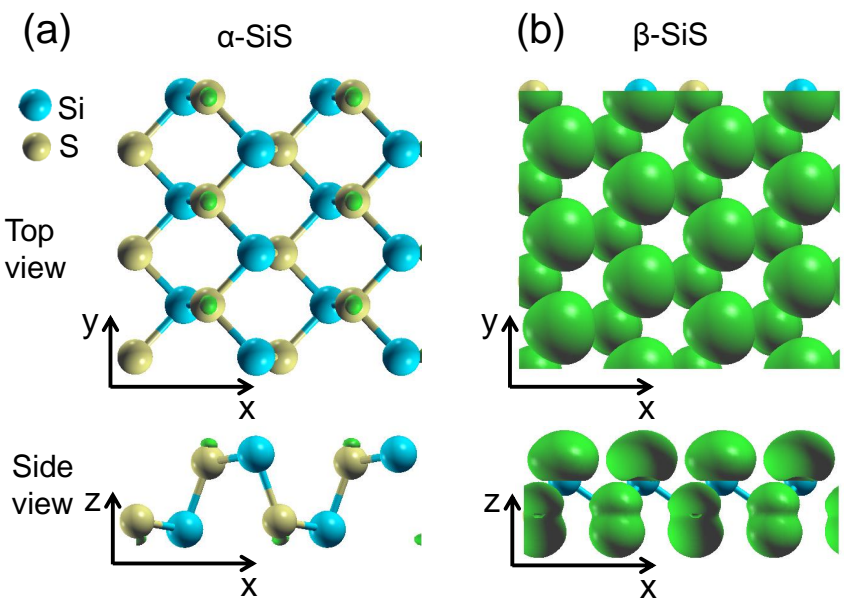

FIG. 2. (Color online) Electron density $\rho_{v b}$ associated with states in the energy range between the Fermi level $E_{F}$ and $0.2 \mathrm{eV}$ below the top of the valence band, shown by the shaded region in Fig. 1 in a monolayer of (a) $\alpha$-SiS and (b) $\beta$-SiS. $\rho_{v b}=2.5 \times 10^{-3} \mathrm{e} / \AA^{3}$ contours are superposed with ball-andstick models of related structures.

structure of the valence and the conduction band region in DFT is believed to closely correspond to experimental results. In Fig. 2, we show the charge density associated with frontier states near the top of the valence band. These states, which correspond to the energy range highlighted by the green shading in the band structure of $\alpha$-SiS in Fig. 1(b) and that of $\beta$-SiS in Fig. 1(e), cover the energy range between the mid-gap and $0.2 \mathrm{eV}$ below the top of the valence band. The valence frontier states of $\alpha$-SiS in Fig. 2(a) and $\beta$-SiS in Fig. 2(b) are similar in spite of the notable charge density differences caused by the larger DOS of $\beta$-SiS in this energy range. The difference between $\mathrm{S}$ and $\mathrm{Si}$ atoms is also reflected in the character of the valence frontier states at these sites. Whereas $p_{z}$ states contribute most at sulfur sites, silicon sites contribute a mixture of $s$ and $p_{z}$ states. These frontier states differ from those of phosphorene, which are related to lone pair electron states.

Similar to phosphorene, the fundamental band gap value of $\mathrm{SiS}$ also depends sensitively on the in-layer strain, as seen in Fig. 3. Due to their nonplanarity, accordion-like in-layer stretching or compression of SiS structures may be achieved at little energy cost as shown in the Supporting Information. The energy cost is particularly low for a deformation along the soft $x$-direction, requiring $\lesssim 20 \mathrm{meV} /$ atom to induce a $\pm 9 \%$ in-layer strain. We believe that in view of the softness of the structure, similar strain values may be achieved during epitaxial growth on particular incommensurate substrates. We also note that tensile strain values such as these have been achieved experimentally in suspended graphene membranes that are much more resilient to stretching due to their planar geometry and stronger bonds 15 17. Con- sequently, we believe that strain engineering is a viable way to effectively tune the fundamental band gap in these systems.

Our results for $\alpha$-SiS in Fig. 3(a) indicate that the band gap decreases when the structure is compressed and increases slightly when it is stretched. The largest change in the band gap, namely its reduction to $0.3 \mathrm{eV}$, may be achieved during a $9 \%$ compression. As seen in Fig. 33(b), we expect the fundamental band gap of $\beta$-SiS to be reduced during both stretching and compression. Within the $\pm 9 \%$ range, we find that the band gap may be tuned in the range from $\approx 1.0-2.3 \mathrm{eV}$. This high degree of band gap tunability in SiS appears particularly attractive for potential applications in flexible electronics.

Since the geometry and lattice constants of SiS and phosphorene are very similar, it is likely that the two could interface naturally in lateral and vertical heterostructures, thus further advancing the tunability of their electronic properties. In Fig 4 4 we present electronic structure results for bilayers consisting of an SiS and a phosphorene monolayer in both $\alpha$ - and $\beta$-phases as the simplest examples of vertical heterostructures. We have optimized the bilayer structures assuming commensurability, i.e. setting the primitive unit cells of each monolayer to be the same. The optimum geometry of the $\alpha-(\mathrm{SiS} / \mathrm{P})$ bilayer is shown in Fig 4 (a) and that of the $\beta$-(SiS/P) bilayer in Fig $4(\mathrm{~b})$.

We find the inter-layer interaction in the two bilayer systems to be rather weak, amounting to $\lesssim 20 \mathrm{meV}$ /atom based on our DFT-PBE calculations. Taking better account of van der Waals interactions would likely increase the calculated interaction energy and reduce the large interlayer separation of $d \lesssim 5.4 \AA$. Whereas the precise interlayer interaction and separation are not of primary concern here, our most important finding is that the weak interaction is not purely dispersive in nature, since we find a substantial rehybridization of states between the adjacent SiS and phosphorene layers. Consequently, as we show more explicitly in the Supporting Information, the bilayer band structure is not a mere superposition of the two monolayer band structures in the same assumed geometry.

As shown in Fig. 4 (b), the $\alpha-(\mathrm{SiS} / \mathrm{P})$ bilayer is a directgap semiconductor with $E_{g} \approx 0.8 \mathrm{eV}$, smaller than the band gap in either isolated monolayer. Since the gap is indirect in the $\alpha$-SiS monolayer, whereas it is direct in $\alpha-\mathrm{P}$, the cause for the direct band gap in $\alpha-\mathrm{SiS} / \mathrm{P}$ may be the dominance of $\mathrm{P}$ states near the gap. We find this conjecture confirmed by examining the frontier orbitals of the bilayer. As we show in the Supporting Information, the frontier states in the conduction band region of the bilayer are essentially purely P-based, whereas those in the valence band region have only a small contribution from the SiS layer.

The electronic band structure of the AA-stacked $\beta$ $(\mathrm{SiS} / \mathrm{P})$ bilayer is presented in Fig. 4(d). The funda- 

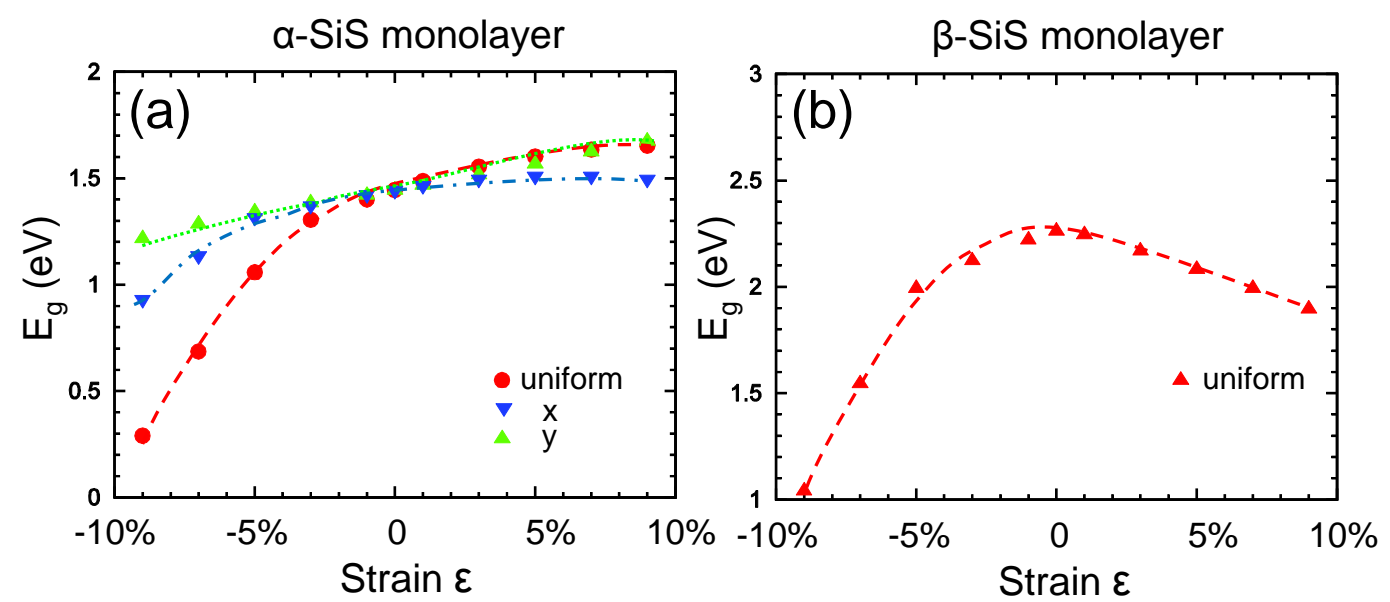

FIG. 3. (Color online) Electronic band gap of (a) $\alpha$-SiS and (b) $\beta$-SiS monolayers as a function of the in-layer strain. Besides the uniform strain, we also show results for uniaxial strain along the $x$ and $y$ direction in the anisotropic structure of $\alpha$-SiS in (a). The lines are guides to the eye.

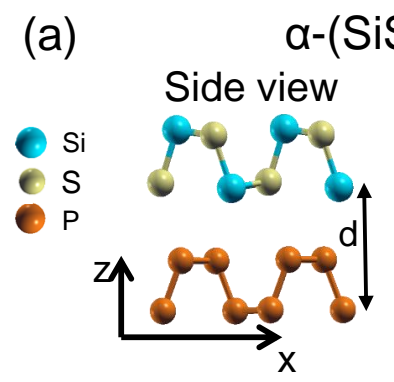

(b)

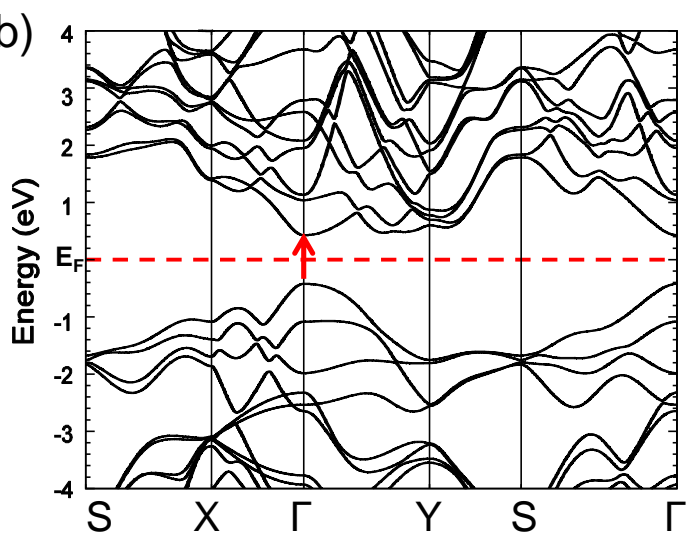

(c)

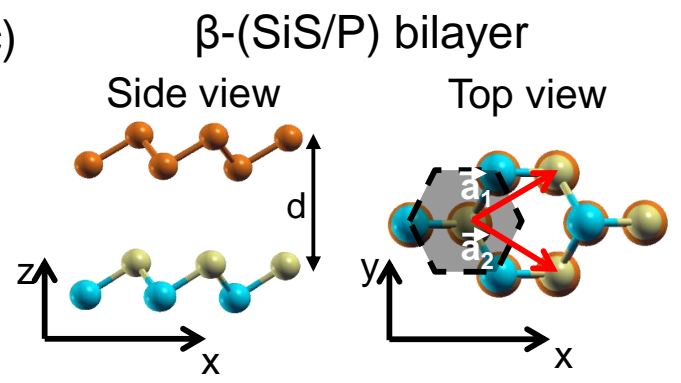

(d)

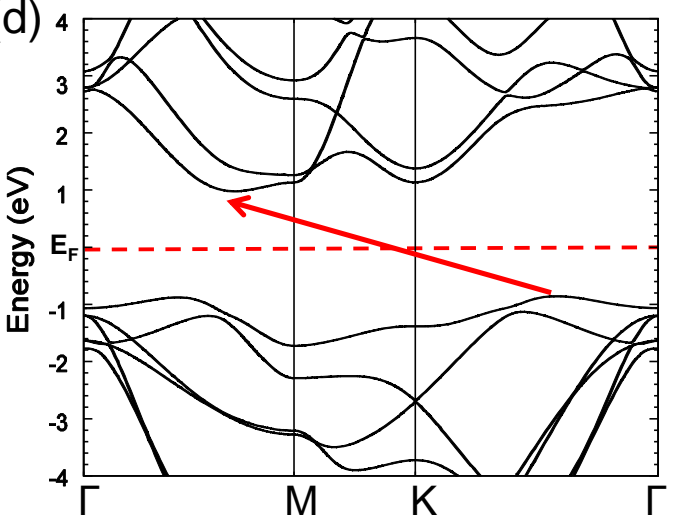

FIG. 4. (Color online) Optimum geometry and electronic band structure of [(a) and (b)] an $\alpha$-(SiS/P) bilayer and [(c) and (d)] a $\beta$-(SiS/P) bilayer. The optimum stacking of the SiS and the phosphorene monolayers in the $\alpha$ - $(\mathrm{SiS} / \mathrm{P})$ bilayer in $(\mathrm{a})$ is $\mathrm{AB}$ and that in the $\beta$-(SiS/P) bilayer is AA.

mental band gap is indirect in this system and its value $E_{g} \approx 1.7 \mathrm{eV}$ is again smaller than in isolated blue phosphorene and $\beta$-SiS monolayers. As seen in the Supporting Information, also the frontier states of $\beta$-(SiS/P) are dominated by $\mathrm{P}$ orbitals both in the valence and the conduction band region.

Since both SiS and phosphorene are rather flexible, they may adjust to each other and form also in-layer heterostructures at little or no energy penalty. We con- structed two types of $\mathrm{SiSP}_{2}$ heterostructures for both $\alpha$ and $\beta$ allotropes and show their geometry and electronic structure in Fig. 5. One type of heterostructures, shown in Fig. 5(a) and 5(e), contains P-P and Si-S atom pairs completely separated from like atom pairs. The other type of heterostructures, presented in Fig. 5(c) and $5(\mathrm{~g})$, contains alternating, contiguous SiS and phosphorus chains. These structures maintain a rectangular lattice with four atoms per unit cell. Generally, we 

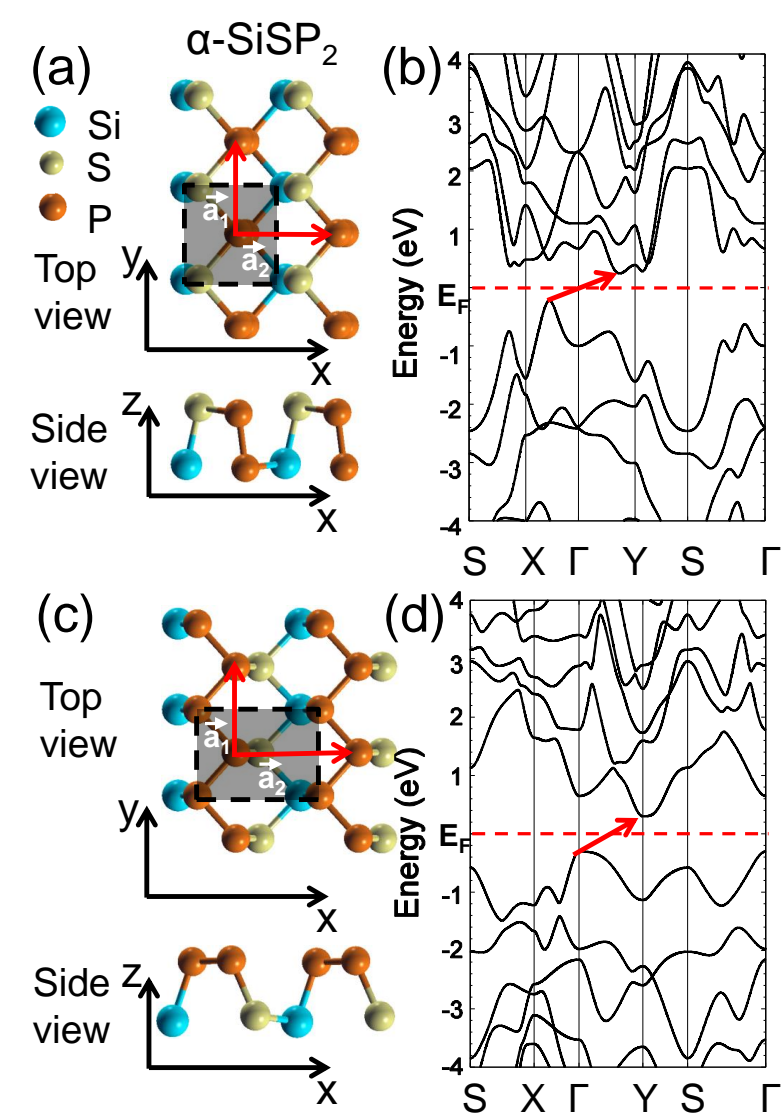

(d)

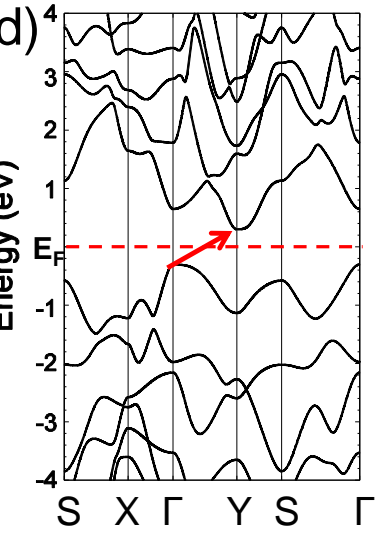

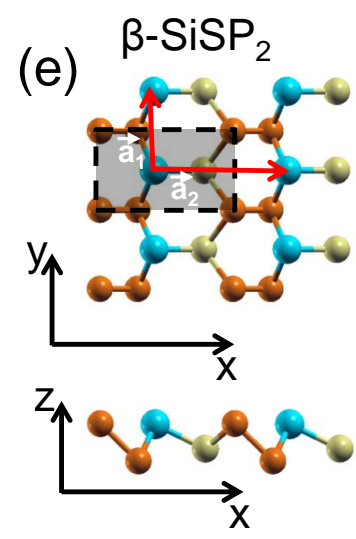

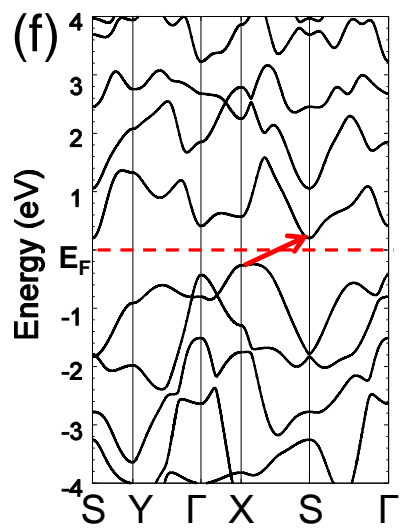

(g)
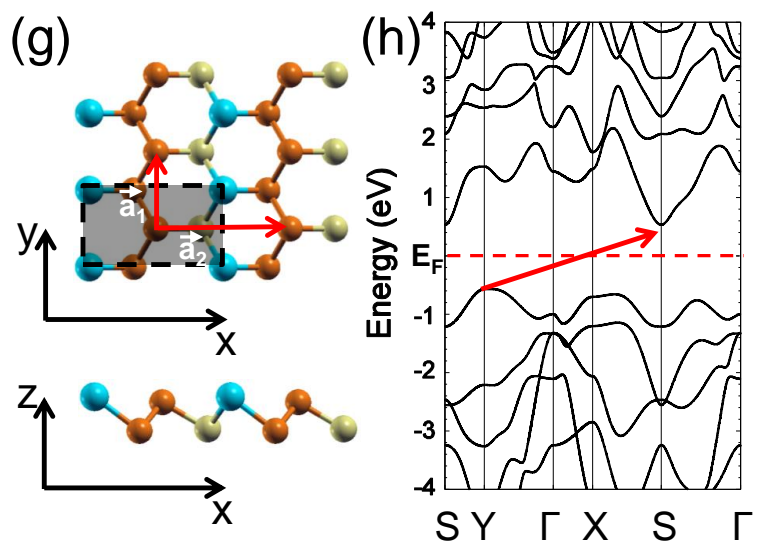

FIG. 5. (Color online) Optimum geometry and electronic band structure of [(a) to (d)] two different in-layer heterostructures of $\alpha$-SiSP 2 and [(e) to (h)] two different in-layer heterostructures of $\beta$-SiSP 2 . The heterostructures differ in the arrangement of $\mathrm{P}$ atoms.

found the in-layer heterostructures to be less stable than pure phosphorene and SiS monolayers. The least stable heterostructures among these are those with isolated P-P or Si-S atom pairs, shown in Fig. 5(a) and 5(e), which are $\approx 0.2 \mathrm{eV} /$ atom less stable than SiS and phosphorene monolayers due to their highly frustrated geometries. The heterostructures with contiguous phosphorus and SiS chains, presented in Fig. 5(c) and 5(g), may better optimize the nearest-neighbor environment. This causes less frustration, making these systems only $\approx 0.1 \mathrm{eV} /$ atom less stable than isolated SiS and phosphorene monolayers.

We found all four in-layer heterostructures to be indirect-gap semiconductors. As in the vertical heterostructures, we found the fundamental band gaps to be substantially smaller than in isolated $\mathrm{SiS}$ and phosphorene monolayers. As seen in Fig. 5(a,b) and 5(e,f), the fundamental band gap $E_{g}$ is close to $0.5 \mathrm{eV}$ in the less stable heterostructures with isolated $\mathrm{Si}-\mathrm{S}$ and P-P pairs. We found larger band gap values in the more stable heterostructures with contiguous $\mathrm{SiS}$ and $\mathrm{P}$ chains, namely $E_{g} \approx 0.6 \mathrm{eV}$ in $\alpha$-SiSP 2 shown in Fig. $5(\mathrm{c}, \mathrm{d})$ and $E_{g} \approx 1.1 \mathrm{eV}$ in $\beta$-SiSP 2 shown in Fig. 5 (g,h), both nearly half the value of the smaller band gap in either SiS or phosphorene monolayers. These findings indicate an intriguing possibility of isoelectronic doping as an effective way to tune the electronic properties of $\mathrm{SiSP}_{n}$ systems.

In view of the different successful synthesis approaches used to form layered IV-VI compounds such as SiS[13] or $\mathrm{SnS}[18$ with a structure similar to $\alpha$-SiS, we believe that a suitable synthesis path will be found to form also the allotropes introduced in this study. The weak inter-layer interaction in layered pure $\mathrm{SiS}$ compounds or $\mathrm{SiS} / \mathrm{P}$ heterostructures should allow for a mechanical exfoliation yielding monolayers and bilayers, same as in graphene and phosphorene. Chemical Vapor Deposition (CVD), which had been used successfully in the past to grow graphene [19, 20] and silicene [21, may become ultimately the most common approach to grow few-layer SiS on specific substrates.

As suggested above, compounds that are isoelectronic to group $\mathrm{V}$ layered semiconductors are not limited to pure IV-VI systems, but may contain other group V elements in the same layer or in a vertical few-layer heterostructure. The initial interest in black phosphorene could thus be significantly expanded by considering the whole range 
of group IV elements including $\mathrm{Si}, \mathrm{Ge}, \mathrm{Sn}$, and $\mathrm{Pb}$, group VI elements such as $\mathrm{S}, \mathrm{Se}$, and Te, and group V elements such as $\mathrm{P}, \mathrm{As}, \mathrm{Sb}$ and $\mathrm{Bi}$, leading to a virtually limitless number of compounds and structural phases. The large family of IV-VI systems that are isoelectronic to group $\mathrm{V}$ elemental semiconductors will include 2D semiconductors with a sizeable fundamental band gap, a high carrier mobility and chemical stability. Expanding this family to heterostructures containing also group V elements should provide a way to effectively tune the electronic properties of the pristine structures, which will likely bring unlimited richness to the field of $2 \mathrm{D}$ semiconductors.

In conclusion, we have proposed IV-VI compounds as isoelectronic counterparts to layered group V semiconductors in analogy to III-V compounds, which have significantly broadened the scope of group IV semiconductors. Using $a b$ initio density functional theory, we have identified yet unrealized structural phases of silicon mono-sulfide (SiS) including the black-phosphorus-like $\alpha$ SiS and the almost equally stable, blue-phosphorus-like $\beta$-SiS. We found that both $\alpha$-SiS and $\beta$-SiS monolayers display a significant, indirect band gap that depends sensitively on the in-layer strain. Unlike 2D semiconductors of group $\mathrm{V}$ elements with the corresponding nonplanar structure, different SiS allotropes are polar. We found that SiS may form both lateral and vertical heterostructures with phosphorene at a very small energy penalty, offering an unprecedented tunability in structural and electronic properties of SiS-P compounds. Combining other group IV and group VI elements with group V elements is expected to lead to a large family of layered semiconductors with an unprecedented richness in structural and electronic properties.

\section{METHODS}

Our computational approach to study the equilibrium structure, stability and electronic properties of SiS structures is based on $a b$ initio density functional theory with the Perdew-Burke-Ernzerhof (PBE) 12 exchangecorrelation functional as implemented in the SIESTA [22] and VASP 23 26 codes. We use periodic boundary conditions throughout the study, with few-layer structures represented by a periodic array of slabs separated by a vacuum region of $\geq 15 \AA$. In our SIESTA calculations we used norm-conserving Troullier-Martins pseudopotentials 27. and a double- $\zeta$ basis including polarization orbitals. The reciprocal space has been sampled by a fine grid 28] of $8 \times 8 \times 1 k$-points in the Brillouin zone of the primitive unit cell of the $2 \mathrm{D}$ structures. We used a mesh cutoff energy of 180 Ry to determine the self-consistent charge density, which provided us with a precision in total energy of $\leq 2 \mathrm{meV} /$ atom. In VASP calculations, we used an energy cut-off of $500 \mathrm{eV}$ and the same k-point sampling as mentioned above. All ge- ometries have been optimized using the conjugate gradient method 29], until none of the residual HellmannFeynman forces exceeded $10^{-2} \mathrm{eV} / \AA$. Equilibrium structures and energies based on SIESTA have been checked against values based on the VASP code.

\section{ACKNOLEDGEMENT}

This study has been supported by the National Science Foundation Cooperative Agreement \#EEC-0832785, titled "NSEC: Center for High-rate Nanomanufacturing". Computational resources have been provided by the Michigan State University High Performance Computing Center.

* tomanek@pa.msu.edu

[1] Liu, H.; Neal, A. T.; Zhu, Z.; Luo, Z.; Xu, X.; Tomanek, D.; Ye, P. D. Phosphorene: An Unexplored 2D Semiconductor with a High Hole Mobility. ACS Nano 2014, 8, 4033-4041.

[2] Zhu, Z.; Tománek, D. Semiconducting Layered Blue Phosphorus: A Computational Study. Phys. Rev. Lett. 2014, 112, 176802.

[3] Guan, J.; Zhu, Z.; Tománek, D. Phase Coexistence and Metal-Insulator Transition in Few-Layer Phosphorene: A Computational Study. Phys. Rev. Lett. 2014, 113, 046804.

[4] Li, L.; Yu, Y.; Ye, G. J.; Ge, Q.; Ou, X.; Wu, H.; Feng, D.; Chen, X. H.; Zhang, Y. Black phosphorus field-effect transistors. Nature Nanotech. 2014, 9, 373-377.

[5] Han, M. Y.; Özyilmaz, B.; Zhang, Y.; Kim, P. Energy band-gap engineering of graphene nanoribbons. Phys. Rev. Lett. 2007, 98, 206805.

[6] Elias, D. C.; Nair, R. R.; Mohiuddin, T. M. G.; Morozov, S. V.; Blake, P.; Halsall, M. P.; Ferrari, A. C.; Boukhvalov, D. W.; Katsnelson, M. I.; Geim, A. K. et al. Control of graphene's properties by reversible hydrogenation: evidence for graphane. Science 2009, 323, 610-613.

[7] Radisavljevic, B.; Radenovic, A.; Brivio, J.; Giacometti, V.; Kis, A. Single-layer MoS2 transistors. Nature Nanotech. 2011, 6, 147-150.

[8] Zhao, L.-D.; Lo, S.-H.; Zhang, Y.; Sun, H.; Tan, G.; Uher, C.; Wolverton, C.; Dravid, V. P.; Kanatzidis, M. G. Ultralow thermal conductivity and high thermoelectric figure of merit in SnSe crystals. Nature 2014, 508, 373377.

[9] Mathews, N. Electrodeposited tin selenide thin films for photovoltaic applications. Solar Energy 2012, 86, 10101016.

[10] Sinsermsuksakul, P.; Heo, J.; Noh, W.; Hock, A. S.; Gordon, R. G. Atomic Layer Deposition of Tin Monosulfide Thin Films. Adv. Energy Mater. 2011, 1, 1116-1125.

[11] Guan, J.; Zhu, Z.; Tománek, D. Tiling Phosphorene. ACS Nano 2014, 8, 12763-12768.

[12] Perdew, J. P.; Burke, K.; Ernzerhof, M. Generalized Gradient Approximation Made Simple. Phys. Rev. Lett. 1996, 77, 3865-3868. 
[13] Hengge, E.; Olbrich, G. Polymere Siliciumsulfide mit Schichtstruktur. Z. anorg. allg. Chemie 1969, 365, 321324.

[14] Tománek, D. Interfacing graphene and related 2D materials with the 3D world. J. Phys.: Condens. Matter 2015, 27, 133203.

[15] Lee, C.; Wei, X.; Kysar, J. W.; Hone, J. Measurement of the Elastic Properties and Intrinsic Strength of Monolayer Graphene. Science 2008, 321, 385-388.

[16] Frank, I. W.; Tanenbaum, D. M.; van der Zande, A. M.; McEuen, P. L. Mechanical properties of suspended graphene sheets. J. Vac. Sci. Techn. B 2007, 25, 25582561.

[17] Huang, M.; Yan, H.; Heinz, T. F.; Hone, J. Probing Strain-Induced Electronic Structure Change in Graphene by Raman Spectroscopy. Nano Lett. 2010, 10, 40744079 .

[18] Su, H.; Xie, Y.; Xiong, Y.; Gao, P.; Qian, Y. Preparation and Morphology Control of Rod-like Nanocrystalline Tin Sulfides via a Simple Ethanol Thermal Route. J. Solid State Chem. 2001, 161, 190 - 196.

[19] Kim, K. S.; Zhao, Y.; Jang, H.; Lee, S. Y.; Kim, J. M.; Kim, K. S.; Ahn, J.-H.; Kim, P.; Choi, J.-Y.; Hong, B. H. Large-scale pattern growth of graphene films for stretchable transparent electrodes. Nano Lett. 2009, 457, 706.

[20] Reina, A.; Jia, X.; Ho, J.; Nezich, D.; Son, H.; Bulovic, V.; Dresselhaus, M. S.; Kong, J. Large area, fewlayer graphene films on arbitrary substrates by chemical vapor deposition. Nano Lett. 2009, 9, 30.
[21] Vogt, P.; De Padova, P.; Quaresima, C.; Avila, J.; Frantzeskakis, E.; Asensio, M. C.; Resta, A.; Ealet, B.; Le Lay, G. Silicene: Compelling Experimental Evidence for Graphenelike Two-Dimensional Silicon. Phys. Rev. Lett. 2012, 108, 155501.

[22] Artacho, E.; Anglada, E.; Dieguez, O.; Gale, J. D.; Garcia, A.; Junquera, J.; Martin, R. M.; Ordejon, P.; Pruneda, J. M.; Sanchez-Portal, D. et al. The SIESTA method; developments and applicability. J. Phys. Cond. Mat. 2008, 20, 064208.

[23] Kresse, G.; Furthmüller, J. Efficient iterative schemes for $a b$ initio total-energy calculations using a plane-wave basis set. Phys. Rev. B 1996, 54, 11169-11186.

[24] Kresse, G.; Hafner, J. Ab initio molecular dynamics for liquid metals. Phys. Rev. B 1993, 47, 558-561.

[25] Kresse, G.; Hafner, J. Ab initio molecular-dynamics simulation of the liquid-metal-amorphous-semiconductor transition in germanium. Phys. Rev. B 1994, 49, 1425114269.

[26] Kresse, G.; Joubert, D. From ultrasoft pseudopotentials to the projector augmented-wave method. Phys. Rev. B 1999, 59, 1758-1775.

[27] Troullier, N.; Martins, J. L. Efficient pseudopotentials for plane-wave calculations. Phys. Rev. B 1991, 43, 1993.

[28] Monkhorst, H. J.; Pack, J. D. Special points for Brillouinzone integrations. Phys. Rev. B 1976, 13, 5188-5192.

[29] Hestenes, M. R.; Stiefel, E. Methods Of Conjugate Gradients For Solving Linear Systems. J. Res. Natl. Bur. Stand. 1952, 49, 409-436. 